Academic Platform Journal of Engineering and Science

journal homepage: $\underline{\text { htp://apjes.com/ }}$

ACADEMIC

$P L A T$ T O R M

\title{
Design of an Android Wear Smartwatch Application as a Wearable Interface to the Diabetes Diary Application
}

\author{
${ }^{* 1,2}$ Ömer Pektaş, ${ }^{2}$ Murat Köseoğlu, ${ }^{3,4}$ Miroslav Muzny, ${ }^{5}$ Gunnar Hartvigsen, ${ }^{3,6}$ Eirik Årsand \\ ${ }^{*}$ Vocational School of Technical Sciences, Electric and Energy, Karamanoglu Mehmetbey University, Karaman, Turkey \\ omerpektas@kmu.edu.tr, \\ ${ }^{2}$ Institute of Science and Technology, Electronics Science, Inonu University, Malatya, Turkey \\ murat.koseoglu@inonu.edu.tr, \\ ${ }^{3}$ Norwegian Centre for E-health Research, University Hospital of North Norway, Tromsø, Norway \\ ${ }^{4}$ Charles University in Prague, 1st Faculty of Medicine, Prague, Czech Republic \\ mmuzny@gmail.com, \\ ${ }^{5}$ UiT The Arctic University of Norway, Department of Computer Science, Tromsø, Norway \\ gunnar.hartvigsen@uit.no, \\ ${ }^{6}$ UiT The Arctic University of Norway, Department of Clinical Medicine, Tromsø, Norway \\ eirik.arsand@ehealthresearch.no,
}

Research Paper

Arrival Date: 17.12 .2019

Accepted Date: 16.11 .2020

\begin{abstract}
In this study, an application was developed for Android-based smartwatches which has the capacity of monitoring the state of diabetes mellitus and indicating the data concerning the physical activities and cardiac rhythm. Android Studio was used to develop and design the application. The application consists of five pages (glucose, insulin, carbohydrate, physical activity, and heart rate) and a watch face. The Dexcom G4 Platinum sensor was used to provide the user's continuous glucose data. The application not only provides monitoring but also allows the users to enter data entry from the pages. Thus, it is possible to use it as a diary by people with diabetes. The development process of the application was done in collaboration with the Norwegian Centre for e-Health Research in Tromsø, Norway. Also, the application operates simultaneously with an Android phone application called Diabetes Diary, which is developed by this research center.
\end{abstract}

Keywords: Diabetes, blood glucose, glucose monitoring, wearable devices, smartwatches, Android, e-Health, wearable sensor.

\section{INTRODUCTION}

Chronic diseases are conditions that usually have slow progress, treated by specific medical interventions and require long-term and periodic follow-up care support to reduce the severity of the disease and to maximize patient's responsibility in self-care [1]. According to the World Health Organization's (WHO) records, chronic diseases are responsible for 63 percent of deaths worldwide. Diabetes mellitus is one of the important chronic diseases affecting approx. 422 million people, and if the tendency continues, it is expected that diabetes will be seventh among causes of death [2].

Diabetes is a chronic disease that is caused by an increase or decrease in the glucose level in the blood.[3]. It is caused by the lack of insulin and/or insufficient secretion of insulin hormone in the blood. Insulin is a hormone that balances the glucose ratio in the blood. It is secreted by pancreas beta cells, and it controls the energy balance of the body [4].
Diabetes is primarily characterized by poorly controlled blood glucose concentrations that, if allowed to remain chronically high, resulting in the development of serious and life-threatening diseases such as stroke, heart attack, heart failure, kidney failure, adult blindness, and amputation [5]. The self-measurement of glucose level at home is an inexpensive control method which is very important for people with diabetes, in order to detect and avoid glucose excursions (high and low values) and to avoid complications of the disease. The glucose monitoring at home is an effective method that can reduce the follow-up and treatment costs of diabetes in the short and long term.

Glucose level measurement is generally performed in two ways, invasive and non-invasive. Invasive measurements use directly blood to measure the amount of glucose while noninvasive one uses alternative biological fluids such as interstitial fluid, saliva, breath, sweat and ocular fluid. Continuous glucose monitoring (CGM) has been shown in studies to be the most effective way to improve glucose control, whether used with insulin injections or a continuous 
insulin pump [6]. The CGM systems use the non-invasive way to monitor the glucose value. CGM measures the glucose level in the interstitial fluid every 1 to 5 minutes from a sensor inserted into subcutaneous tissue. [7]. The CGM sensors use glucose oxidase enzyme which reacts with glucose to provide hydrogen peroxide as a by-product. The reaction between hydrogen peroxide and platinum causes an electrical signal that is transmitted through a thin wire. Afterwards, this signal is taken by CGM system software to converted to a glucose value [8].

Mobile phones, smartwatches and some wearable technologies are used in monitoring the glucose level by processing and presenting the information obtained from the sensor in CGM systems. The sensitivity, reliability, long term availability, biocompatibility, durability and costefficiency are important factors in choosing the proper sensor. There are a few different companies producing sensors intended for CGM systems. In this study, the glucose level measurements were performed by using the Dexcom G4 sensor (Dexcom Inc., California, USA). Various smartphone platforms have been used by big players such as Apple, Samsung, Huawei, etc. The use of these new platforms brings several advantages: the wide varieties of models, the diversity of manufacturers and the integration of various sensors thanks to wireless protocols (Bluetooth, Wi$\mathrm{Fi}$ ), based on the user's own personal needs. Updates in software and hardware allow applications with advanced functions and user interfaces $[9,10]$. The wearables market is growing rapidly, as signified by the rise of worldwide forecasted to increase from 593 million devices in 2018 to 929 million devices by 2021 [11]. Smartwatches have a similar potential to support health in everyday life. These devices can provide meaningful and reliable physical activity data that may have the potential to present new disease management approaches to patients diagnosed with diabetes when used appropriately. In this study, an Android wear application has been designed and developed for managing blood glucose, insulin and carbohydrate intakes. It is also monitoring physical activity (steps) continuously and instant heart rate. In addition, the application has a watch face page to monitor all values at the same time, including time and date. Android Wear devices provide advanced capabilities for watch faces that you can leverage in designs, such as vibrant colors, dynamic backgrounds and data integration [12]. Thus, we decided to design a watch face which is integrated with the application to show all the latest values.

\section{MATERIALS AND METHODS}

\subsection{Materials}

\subsubsection{Android-based smartphone}

Android is a Linux-based open source operating system which has been developed for mobile devices by Google and Open Handset Alliance.
Due to the advancement in wireless communication, effective applications can be developed to make self-control more efficiently with the beneficial feedbacks on diabetes mellitus [13]. The latest generation of smartphones has powerful computing capabilities that enable open application development. This kind of environment allows fast collection of data from internal and external sensors to mobile phones [14].

The developed smartwatch application works synchronized with the Diabetes Diary application which is developed by the Norwegian Center for e-Health Research in Tromsø, Norway (see Fig. 1). Thus, the Android smartphone provides a Bluetooth-based data transfer unit that sends the data to the smartwatch. This application has been developed in order to better control the disease status of patients with diabetes. In addition to easily identifying targets for better quality and healthy life, the user can enter the values of insulin intake, daily physical activity value (step count, running distance, bicycle, etc.), carbohydrate intake and blood sugar values by the user. Thus, the application can be used as a diary [15].
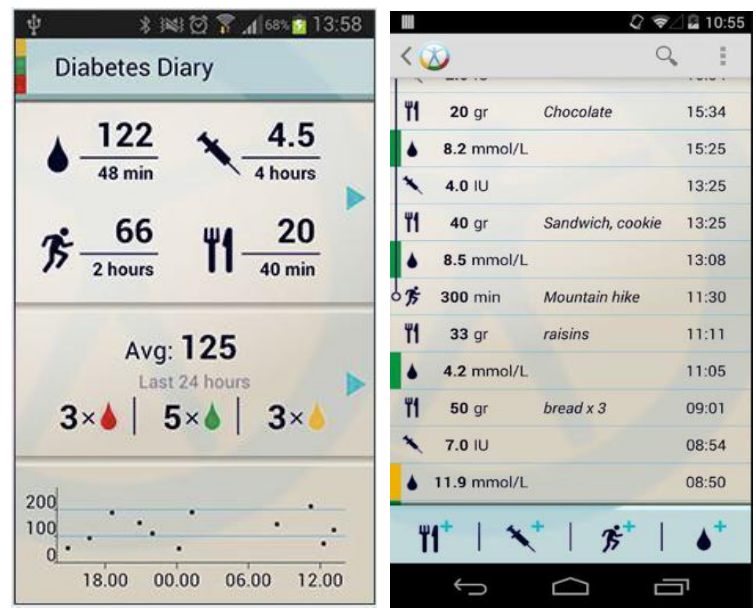

Fig. 1. Diabetes Diary Application [15]

\subsubsection{Android-based smartwatch}

Smartwatches are portable devices which have been designed to be worn on the wrist. In generally, there are two types of smart watches in the wearable product market. First, a general-purpose smartwatch like the Apple Watch and most Google-powered Android Wear OS devices with various forms and functions. They are designed to replace mechanical wristwatches and are heavily smartphone dependent. They use touchscreens, offer apps, and often record some vital signs. Major types include Apple Watch, Samsung Gear S models and Huawei Smartwatch models. The other type includes specialty devices intended for specific use cases. These devices often offer a more powerful version of a fitness tracker and a stand-alone fitness tracker like a Fitbit [16]. 
Huawei Smartwatch 2 has been used to test the application (see Fig. 2) due to high quality integrated sensors which are one of the best on its area according to the user interpretations in exercise applications and the possibility of using without the phone. In addition, it is possible to get notifications, make daily plans and download many applications from its market on the smartwatch. The daily exercises monitoring would be made without need of a mobile phone. Thanks to the integrated sensors, physical activity values (step, running, climbing, etc.) are recorded automatically and continuously monitored [17]. The application is not only designed for this model, but it can also be easily used in any Android-based smartwatch models.
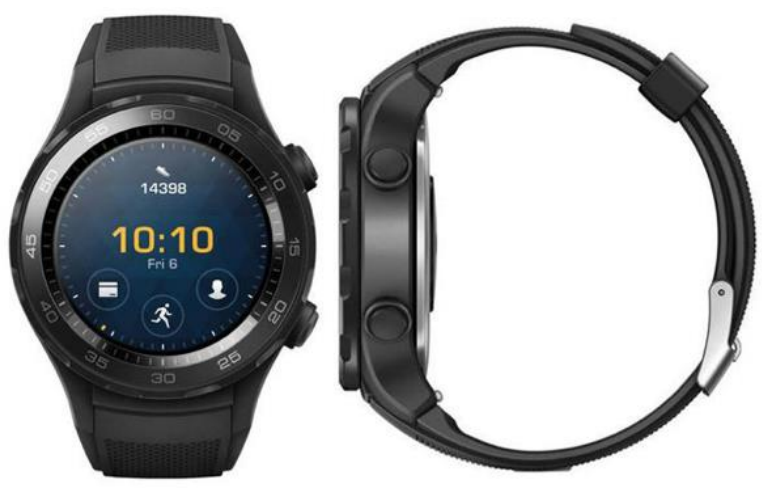

Fig. 2. Huawei Watch 2 [17]

\subsubsection{Dexcom G4 Platinum Sensor}

Dexcom G4 platinum sensor has been used for glucose measurement. Dexcom G4 uses a CGM technology system for measurement, helping to keep your blood sugar within the target range. This can be continuously monitored from the screen without the need of additional processing. The CGM system allows monitoring of glucose levels and trends throughout the day and night. Dexcom G4 can be used by both children and adults, e.g., it can be used safely by parents who want to provide more effective control for their children with Type 1 (or Type 2 diabetes) [18].

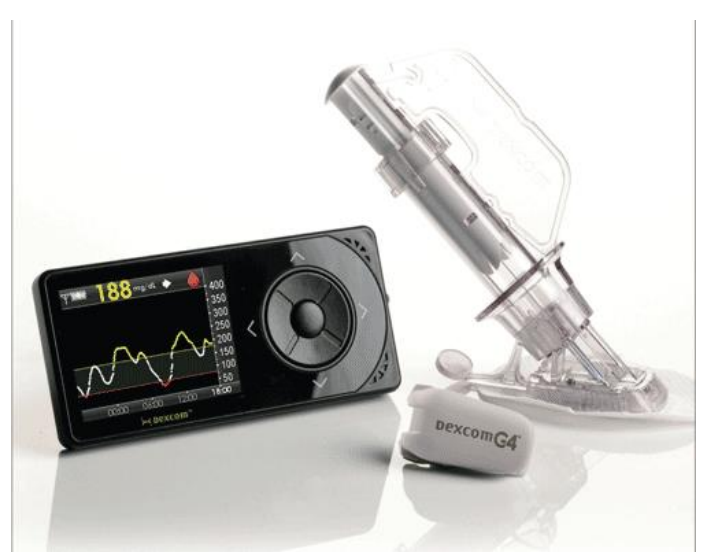

Fig. 3. Dexcom G4 Platinum [18]
The Dexcom CGM helps to know your glucose number and where it's headed and without finger sticks or calibrations required. [14]. The Dexcom G4 Platinum consists of three parts: transmitter, receiver, and sensor.

In this project, we have used a mobile phone as a receiver instead of the Dexcom receiver. This means that we only used the sensor and transmitter to measure and send the glucose data.

\subsection{4. $\quad$ Drip Module}

xDrip module acts as a bridge between the Dexcom G4 Transmitter and a Bluetooth Low Energy device such as a mobile phone (see Fig 4). The data from a Dexcom G4 transmitter package has been received by the programmable radio through Wixel and transferred to the phone via Bluetooth.

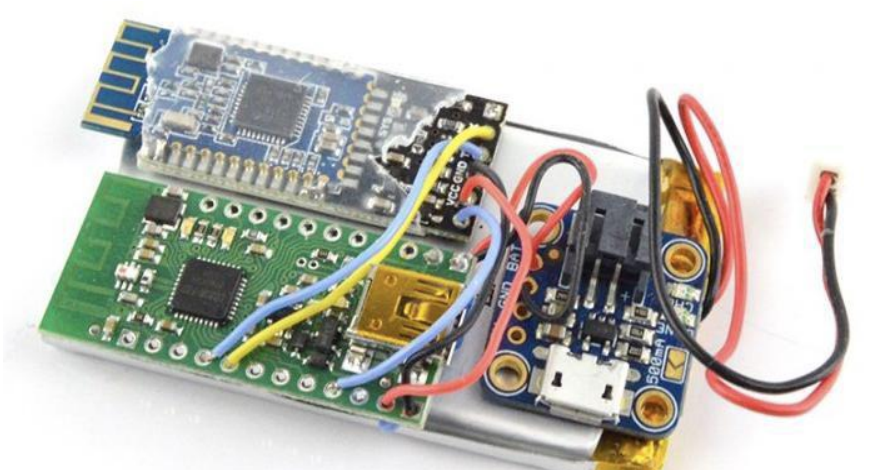

Fig. 4. xDrip Module

Only three pieces of data are received from the Dexcom G4. Two of these are 32-bit glucose values and the other is an 8bit value that indicates the battery status of the Dexcom G4. The xDrip circuit diagram with the HM-10 BLE module is shown in Fig. 5.

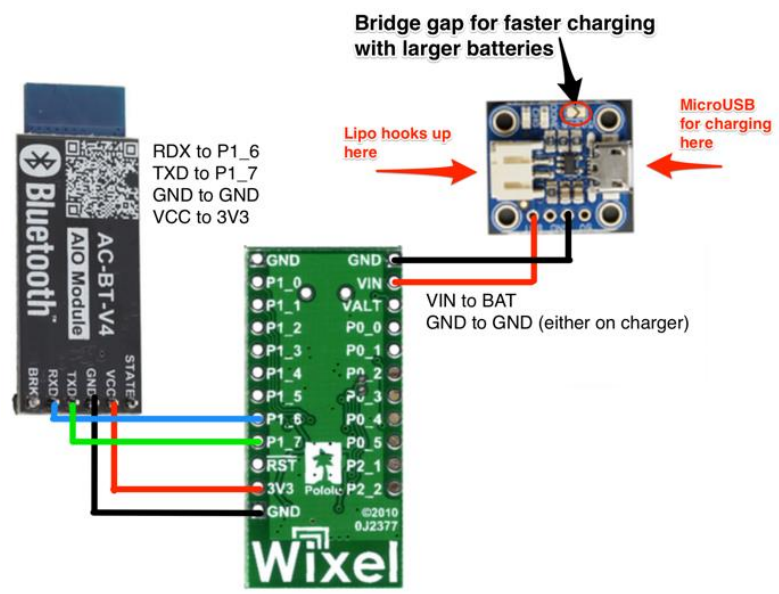

Fig. 5. xDrip Circuit with HM-10 BLE module [20] 


\subsection{Method}

The data read by the Dexcom glucose sensor can be transmitted to the telephone by a transmitter via the xDrip bridge module and software. But this is not standard and based on Nightscout project which is an open-source, DIY (do it yourself) spearheading that allows real-time access to CGM data through smart devices [21]. This process is controlled by the xDrip application installed on the phone. The application allows the module to connect to the Dexcom sensor via radio frequency (RF). When the connection is provided between the sensor and mobile phone, the glucose data is transmitted via Bluetooth to the application, and it is displayed in the smartwatch application simultaneously, due to the two-way synchronization.

The diagram in Fig. 6 shows the algorithm that represents the way of data from the measurement point to the application developed in this study. Here, the data is taken to the phone via the $\mathrm{xDrip}$ application and sent to the server with the same application. Data from the server with the Diabetes Diary application is transferred via the phone to the smartwatch application.

The developed Android smartwatch application retrieves the data from the Diabetes Diary application connected to the Nightscout server. In other words, the developed application is an Android smartwatch version of the Diabetes Diary diabetes smartphone application. When the phone is paired with the smartwatch, both applications work through twoway synchronization. In addition, the values can be used as a diary by entering the values from the application. The application was developed by using the Java programming language on Android Studio which is the official development environment for Android devices.

It is always important to get the opinion of people who have diabetes mellitus before designing the application. Because the design purpose of the application is to provide ease of use to these people and increase their motivation to have a positive impact on their quality of life. For this reason, some meetings were held every other week with the people with diabetes who work in the research center, and ideas were exchanged during the pre-design and design period.

\subsubsection{Android Studio}

Android Studio is an official integrated development environment (IDE) for Android operating system. Introduced in the Google I / O event on May 16, 2013, and is based on IntelliJ IDEA. It was designed specifically for Android development. Applications for phone, tablet and wearable devices (watch, glasses, etc.) can be developed through Android Studio. Android applications are written in the Java programming language. In addition, since 2017, the Kotlin programming language for the Android platform has been supported [22].
In the project, the application was developed on Google's Wear OS platform for Google's wearable smartwatches in the Android Studio interface; some of Google's libraries were used. These are the Android Wear SDK for wearable devices, Google Play Services for receiving data from Google applications, Google's DataClient for the communication between devices, SensorEventListener to read values from integrated sensors within the smartwatch, and ViewPager libraries to switch between pages.

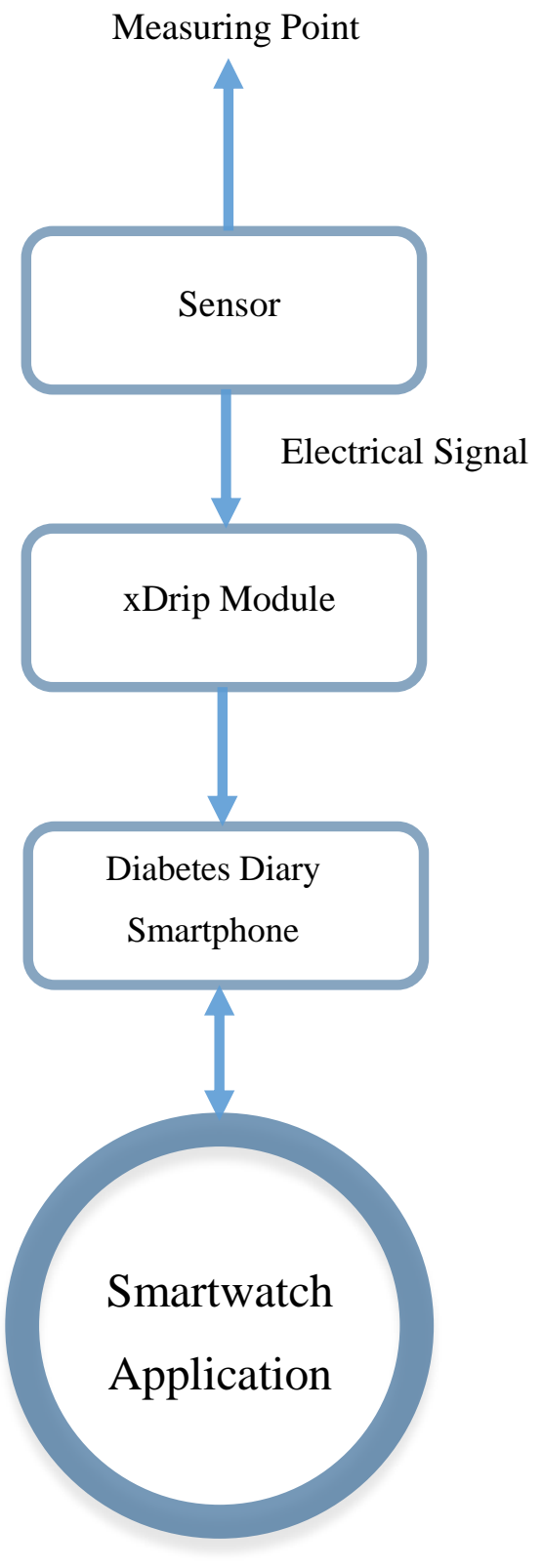

Fig. 6. Illustration of System components

\section{RESULTS}

In this project, Android-based smartphone and smartwatch, Dexcom G4 Platinum sensor and xDrip/Nightscout data transfer circuit have been used for glucose measurement. The 
measurement was made by the sensor, and glucose data was transferred to the phone or watch via Bluetooth. The data has been processed to make it comprehensible for patients by smart devices. Then, the meaningful data has been presented by the application. In this application, totally five pages were designed for each value. Each page had its own specific design and backgrounds; especially on the pages of glucose, insulin and physical activity, the page background changes based on the values. The shift between the pages can be made by sliding to left and right. Apart from these pages, an interface (Watch Face) was designed for monitoring of all the values in real-time.

\subsection{The Application Pages \\ 3.1.1. GLUCOSE}

The glucose page has three different backgrounds. Each background design has its own aim during the monitoring. Based on the status of the glucose value, the user is informed with various colors and faces. Thus, the user can understand own glucose status with the background design without the need to read the value. The aim of this design is to provide comfortable monitoring of glucose status and motivate the user with the alternating graphical user interface (GUI) faces.

If the glucose value is less than or equal to 10 millimole/liter ( $\mathrm{mmol} / \mathrm{L})$, and greater than $4 \mathrm{mmol} / \mathrm{L}$, then the glucose status is in the desired range. Thus, the background turns into a green color and a smiley face (see in Fig. 7a). If the value is greater than 10 millimole/liter $(\mathrm{mmol} / \mathrm{L})$ and less than 20 $\mathrm{mmol} / \mathrm{L}$, the background turns into a yellow color and a normal face (see in Fig. 7b). And, if the value is equal to 4 $\mathrm{mmol} / \mathrm{L}$ or greater than $20 \mathrm{mmol} / \mathrm{L}$, it is an undesirable range that patients pay attention to. Thus, the background turns into a red color and a sad face (see Fig. 7c). Moreover, seeing instant time and date are possible in each background.

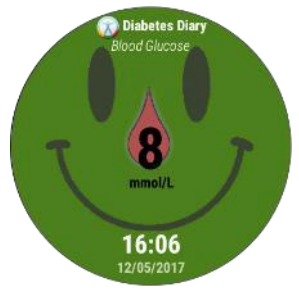

a

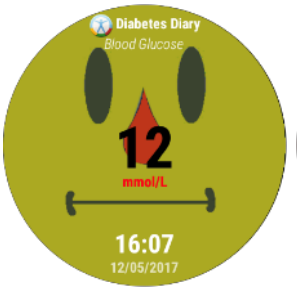

b

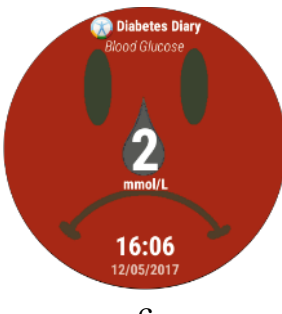

c
Fig. 7. Glucose Page Designs

\subsubsection{Insulin Page}

A hollow injector is located in the middle of the insulin page, and it is filled or discharged depending on the entered injected insulin value. The aim here is to provide the user with a visually better and motivating design. The injector is filled based on the insulin value between 0 and 20 in international units (iu). The page presents to the user this value along with the current date and time (see Fig. 8).
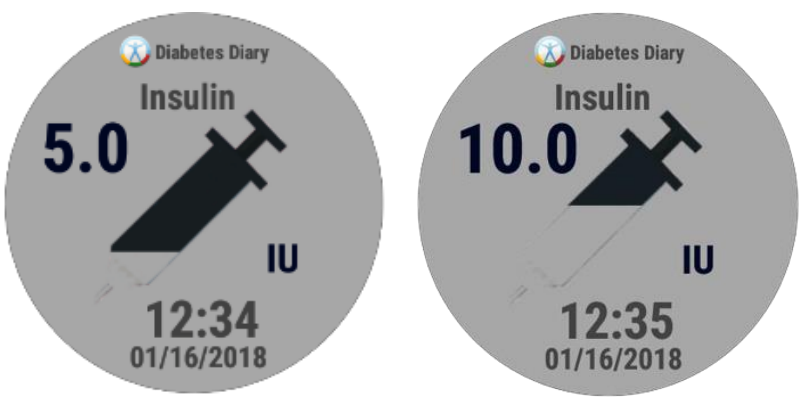

Fig. 8. Insulin Page Design

\subsubsection{Carbohydrate Page}

The carbohydrate page has a single background. The design consists of plates, forks, and spoons. The entered intake of carbohydrate in the middle of the plate pattern can be seen in grams (gr) along with providing instant time and date (see Fig. 9).

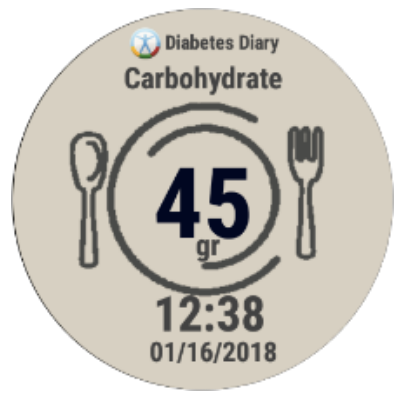

Fig. 9. Carbohydrate Page Design

\subsubsection{Physical Activity Page}

The physical activity page shows the number of steps. The user can also open the number entry screen by tapping the target step number under the "Goals" on this page. Thus, the target number can be entered manually. The maximum value of the progress bar, which is in the shape of a blue circle around the page, changes depending on the specified number of targets. This bar shows how close the user is to his or her target at each step in a visual way. The number of steps is daily, thus it resets every 24 hours. This value is read by the accelerometer sensor which is integrated into the smartwatch (see Fig. 10).

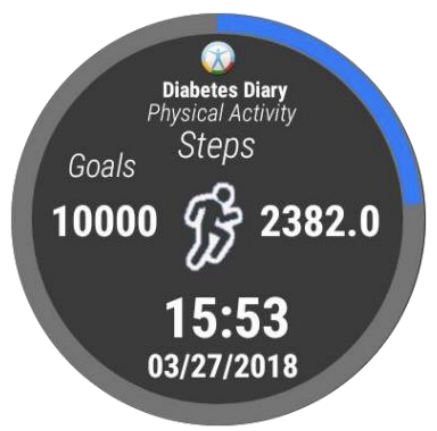

Fig. 10. Physical Activity Page Design 


\subsubsection{Heart Rate Page}

The heart rate value is seen in the middle part of the watch screen, see picture in Fig. 11. This value changes instantly and is taken directly from the integrated sensor in the smartwatch. When the page is opened for heart rate measurement, four points will be displayed first. These points indicate that the measurement is performed, and the heart rate value can be seen in one second (see Fig. 11).
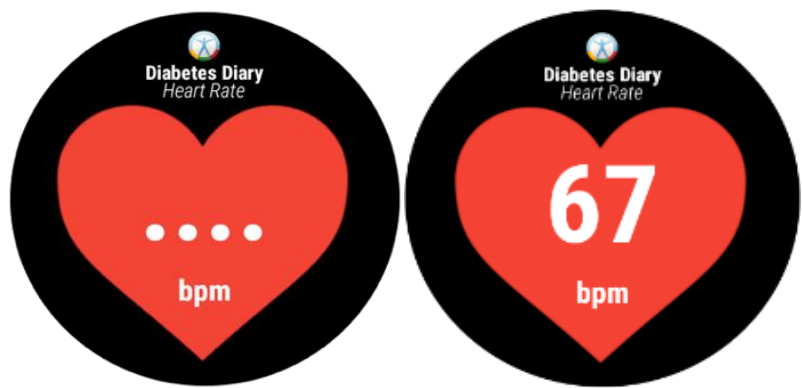

Fig. 11. Heart Rate Page Designs

\subsubsection{Data Entering Page}

This page opens up by touching the values on glucose, insulin and carbohydrate on own pages. On the physical activity page, the data entry page can be reached by touching the number of goals section, which specifies the daily goal. The numbers between 0 and 9, dot, and dashes (-) are displayed as in the calculator style. After entering the desired value, the button with the check mark can be registered. Thus, the value will be entered by the user. Also, the wrong value can be deleted with the backspace button (see Fig. 12).

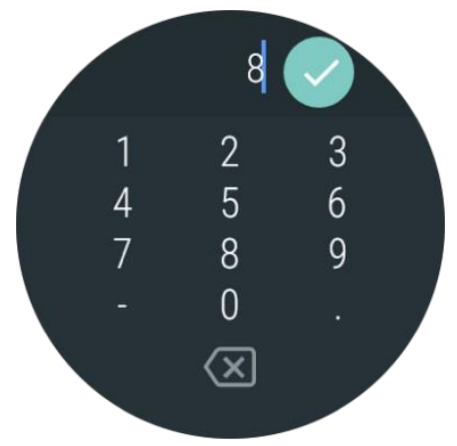

Fig. 12. Data Entering Page Designs

\subsection{Diabetes Diary Android Wear Watch Face}

Here, the glucose portion was designed colorful to indicate the status like on glucose page. The color indicator is varying depending on the glucose values as in the glucose page in the application. Access to the application can also be provided through this interface by touching the values. Each value is linked to its own page, and when the value is touched, the application page of that value is opened (see Fig. 13).

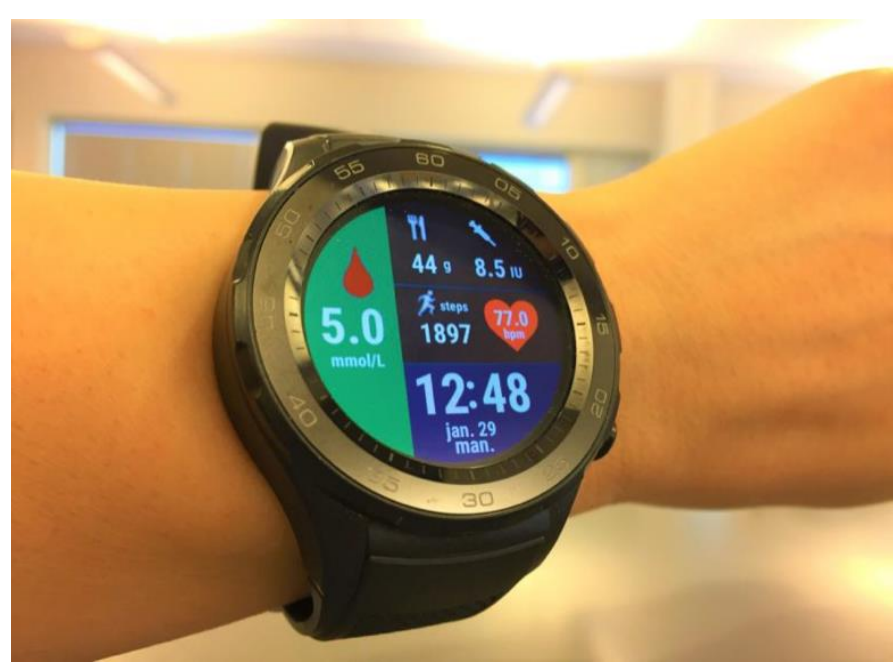

Fig. 13. Diabetes Diary Watch Face 1

One of the most important details added to this interface is the time statement below the values. This statement indicates when the values are last received or changed. As an example, the user is able to learn by checking this time statement how many minutes or hours ago he controlled the glucose status (see Fig. 14).

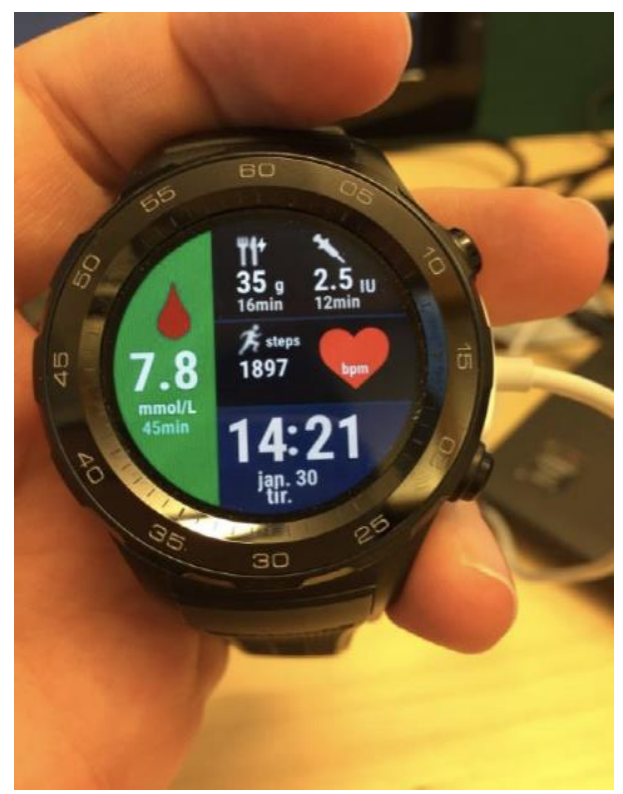

Fig. 14. Diabetes Diary Watch Face 2

\section{DISCUSSION}

The users tested the initial phase of this application with the phone application. The feedbacks were mostly positive. The users found the application easy to use since they did not need to control the phone constantly. The user, who has diabetes mellitus, has reported that he found the application motivating, beneficial and useful due to its colorful interface, warnings and alerts. He also mentioned that value tracking could be done easily. On the other hand, the user, who does not have diabetes mellitus, has stated that the daily 
carbohydrate intake due to the carbohydrate data entry could be monitored by the application easily. He has also denoted that the step counter and heart rate monitoring were also very practical and useful. Both testers expressed that they liked the watch face design since it could be easily followed at any time of the day due to its colorful glucose part, easy and practical screen scroll and accurate measurements. As a drawback, the person with diabetes stated that it would be better if it was connected to the Nightscout server to provide the CGM data.

The Diabetes Diary application also has a version for Pebble smartwatches. The Pebble application was developed by the Norwegian Center for e-Health Research as a Pebble version of Diabetes Diary application in 2014 [23]. The Pebble application is monitoring the glucose, insulin, carbohydrate intake and physical activity values and also working twoway synchronization with the smart phone version of the application (see Fig. 15). However, since Pebble watches no longer are available, new diabetes management apps for smartwatches are needed.

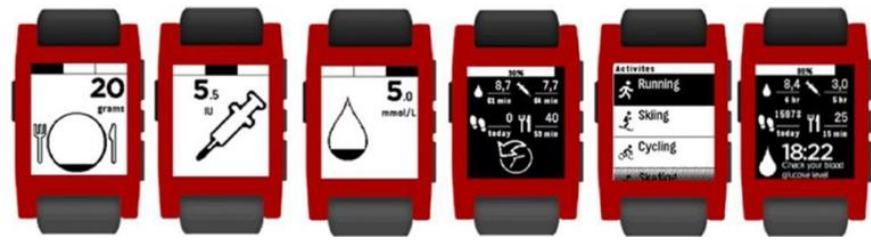

Fig. 15. Diabetes Diary Pebble Application [18]

One of the test users, who was also using the Pebble smartwatches version of "Diabetes Diary" application in his daily life, compared these two applications and stated the advantages of the application developed in this study as follows:

- The area of glucose monitoring is colorful, thus more motivational and easier to control the status,

○ In general, the font size of the data should be easier to read,

- The data rate is faster than the Pebble application,

- Each value is presented more motivating due to its design and color.

Thus, due to these reasons mentioned above, the user concluded that this application is more useful. However, CGM data has not been displayed on the application at this time because the project has not been completed yet. Future work is needed to connect the Nightscout server and visualize glucose at the graphical interface. This was the only feature mentioned from the users as a missing part of this application. We think that the users do not want to stay connected to the phone application for receiving the CGM data from the server, but rather receive it directly on the smartwatch application.
Table 1. Comparison of some diabetes applications with the developed application for smartwatches

\begin{tabular}{cccc}
\hline & $\begin{array}{c}\text { Diabetes:M } \\
\text { Features }\end{array}$ & $\begin{array}{c}\text { Balansio } \\
\text { App }\end{array}$ & $\begin{array}{c}\text { Developed } \\
\text { App }\end{array}$ \\
\hline Glucose & $\checkmark$ & $\checkmark$ & $\checkmark$ \\
Insulin & $\checkmark$ & $\checkmark$ & $\checkmark$ \\
Physical & - & $\checkmark$ & $\checkmark$ \\
Activity & - & - & $\checkmark$ \\
Heart Rate & - & $\checkmark$ & $\checkmark$ \\
$\begin{array}{c}\text { Glucose Data } \\
\text { Input }\end{array}$ & $\checkmark$ & $\checkmark$ & $\checkmark$ \\
$\begin{array}{c}\text { Insulin Data } \\
\text { Input }\end{array}$ & $\checkmark$ & $\checkmark$ & $\checkmark$ \\
Carbohydrate \\
Data Input
\end{tabular}

Also, there are some other applications that present essential data via smartwatches and developed before our application. One of them is Diabetes:M smartwatch application [24]. This application contains some features which are already included by the application developed in this study. These are tracking glucose level, insulin dose, carbohydrate intake and data inputs by user on the watch. The application developed in this study also presents physical activity and heart rate values to the user, unlike the Diabetes:M application. Additionally, Balansio application can be used to present glucose level, insulin dose, carbohydrate intake, physical activity and data inputs [25]. The difference between our application and Balansio application is heart rate measurement. Diabetes: $M$ application, Balansio application and the application developed in this study were presented in Table 1 comparatively.

\section{CONCLUSION}

A smartwatch application was developed for Android-based smartwatches having the capacity of monitoring the state of glucose, insulin, carbohydrate intake, meanwhile, illustrating the data concerning the physical activities and cardiac rhythm. The application was designed on Android Studio by synchronizing with the Diabetes Diary mobile application. Bluetooth communication was used to transfer data, namely glucose, insulin, carbohydrate intake as well as physical activity. Furthermore, the watch face was designed for the smartwatch to make it possible to monitor and control the values in a more convenient way for daily usage than through a mobile phone.

The developed application was tested by the users and found quite useful, beneficial and practical. They denoted that it is motivating with its colorful well-marked faces, warnings and alerts. Also, they have stated some missing features which can be included in the developed application. Additionally, the developed application was compared with some former applications intended for diabetes mellitus, and advantages of developed application presented clearly. The developed 
design serves as a basis for future research and development of a combined diabetes diary application for Android Wear or other smartwatch platforms and smartphones.

\section{FUTURE WORK}

Considering the feedbacks from the users, the application is planned to be integrated to the Nightscout server to benefit from the advantage of direct connection between the application and server.

\section{ACKNOWLEDGMENTS}

This project has been designed and developed at the Norwegian Centre for e-Health Research, Tromsø, Norway. We would like to thank the research center's staff who provided insight and expertise that greatly assisted the project, and provided great hospitality to the first author.

\section{REFERENCES}

[1]. Ü. Özdemir, S. Taşcı. J. Erciyes Üniversitesi Sağlık Bilimleri Fakültesi Dergisi, "Kronik Hastaliklarda Psikososyal Sorunlar ve Bakim," vol. 1, no. 1, pp. 57-72, 2013.

[2]. C. Chen, X.-L. Zhao, Z.-H. Li, Z.-G. Zhu, S.-H. Qian, and A. J. S. Flewitt, "Current and emerging technology for continuous glucose monitoring," vol. 17, no. 1, p. 182, 2017. [3]. Rghioui, Amine, et al. Electronics, "A Smart GlucoseMonitoring System for Diabetic Patient." 9.4 (2020): 678.

[4]. Diyabet, URL: https://hsgm.saglik.gov.tr/tr/diyabet (Accessed on 12

September 2019)

[5]. Masoud, Baghelani, et al. Scientific Reports (Nature Publisher Group) "Non-invasive continuous-time glucose monitoring system using a chipless printable sensor based on split ring microwave resonators." 10.1 (2020).

[6]. Shah, Rajiv, et al. "Multi-analyte continuous glucose monitoring." U.S. Patent Application No. 16/273,920.

[7]. Kim, Jae Hyun. "Current status of continuous glucose monitoring among Korean children and adolescents with type 1 diabetes." Journal of Korean Society of Pediatric Endocrinology (2020).

[8]. M. Koseoglu and O. Pektas, "Smart and Wearable Technologies in Continuous Glucose Monitoring," presented at the International Engineering and Natural Science Conference (IENSC 2018), November 2018, 2018.

[9]. J. Helbostad et al., "Mobile health applications to promote active and healthy ageing," vol. 17, no. 3, p. 622, 2017.

[10]. S. H.-W. Chuah, P. A. Rauschnabel, N. Krey, B.
Nguyen, T. Ramayah, and S. J. C. i. H. B. Lade, "Wearable technologies: The role of usefulness and visibility in smartwatch adoption," vol. 65, pp. 276-284, 2016.

[11]. Mück, Julia E., et al. Trends in biotechnology, "Market and patent analyses of wearables in medicine." 37.6 (2019): 563-566.

[12]. Ö. Pektas, M. Muzny, E. Årsand, G. Hartvigsen, M. Koseoglu, "Utilizing the New Gerenration of Wearable Devices in a Combined Diabetes Diary Application," presented at the The innovative 11th International Conference on Advanced Technologies \& Treatments for Diabetes, Vienna,, Austria, 14-17 Februrary, 2018.

[13]. E. I. Georga, V. C. Protopappas, C. V. Bellos, D. I. J. H. Fotiadis, and Technology, "Wearable systems and mobile applications for diabetes disease management," vol. 4, no. 2, pp. 101-112, 2014.

[14]. T. Szydło, M. J. M. Konieczny, and Microsystems, "Mobile and wearable devices in an open and universal system for remote patient monitoring," vol. 46, pp. 44-54, 2016.

[15]. Diabetes Diary, URL: http://www.diabetesdagboka.no/en/

[16]. S. Silbert. (2019). What is a Smartwatch?, URL: https://www.lifewire.com/an-introduction-to-smart-

watches-3441381.

[17]. Huawei Smartwatch 2, URL: https://consumer.huawei.com/tr/wearables/watch2/.

[18]. J. Šoupal et al., "Comparison of different treatment modalities for type 1 diabetes, including sensor-augmented insulin regimens, in 52 weeks of follow-up: a COMISAIR study," vol. 18, no. 9, pp. 532-538, 2016.

[19]. Dexcom, URL: https://www.dexcom.com/tr-TR.

[20]. xDrip Hardware Setup, URL: https://github.com/StephenBlackWasAlreadyTaken/xDrip/b lob/gh-pages/hardware_setup.md.

[21]. D. Beckman, C.M. Reehorst, A. Henriksen, M. Muzny, E. Årsand, G. Hartvigsen. International Journal of Integrated Care. "Better glucose regulation through enabling groupbased motivational mechanisms in cloud-based solutions like Nightscout." 16(5):S4, 2016. dx.doi.org/10.5334/ijic.2548.

[22]. W. Jackson, Pro android wearables: Building apps for smartwatches. Apress, 2015.

[23]. E. Årsand, M. Muzny, M. Bradway, J. Muzik, G. Hartvigsen. Journal of Diabetes Science and Technology, "Performance of the first combined smartwatch andsmartphone diabetes diary application study," vol. 9, no. 3,pp. 556-563, 2015.

[24]. Diabetes:M,

URL: https://sites.google.com/view/diabetes-muserguide/android-wear.

[25]. Balansio, URL: https://www.balansio.com. 